\title{
REPORT ON THE THEORY OF THE GEOMETRY OF NUMBERS.*
}

\author{
BY PROFESSOR H. F. BLICHFELDT.
}

(Read at the Chicaigo Symposium of the American Mathematical Society, March 28, 1919.)

1. Introduction.-There arises in theory of numbers an important class of problems of a kind best illustrated by an example: Consider the quadratic form $f=a x^{2}+2 b x y+c y^{2}$, $a c-b^{2}>0$. Let the numerical value of the determinant $D=a c-b^{2}$ be given, but not the coefficients $a, b, c$ individually; also let it be specified that the variables $x, y$ must be integers (positive or negative or zero). Under these conditions, what can be predicted as to the least absolute value of $f$, other than $f=0$ ? Designating this value by $[f]$, we have $\dagger$ $[f] \leqq 2 / \sqrt{3 D^{\frac{1}{2}}}$, the extreme limit being reached by the form $x^{2}+x y+y^{2}$.

Isolated problems of like nature were studied by prominent mathematicians during the past century. Hermite discovered a superior limit to the least value $[f]$ of a positive definite quadratic form in $n$ variables in terms of $n$ and the numerical value of the determinant $D:[f] \leqq\left(\frac{4}{3}\right)^{(n-1) / 2} D^{1 / n}$ (Journal für Mathematik, volume 40, 1850, page 263); this was the first important result of a general nature.

In the matter of references we shall use the abbreviations: $\mathrm{M}_{1}, \mathrm{M}_{2}, \mathrm{M}_{3}, \mathrm{M}_{4}$ designate respectively the following books by

Minkowski: "Diophantische Approximationen," Leipzig,

1907; "Geometrie der Zahlen," Leipzig, 1896-1910; "Gesammelte Abhandlungen," volumes 1 and 2, Leipzig, 1911.

$\mathrm{B}_{1}$ refers to "A new principle in the geometry of numbers" by the author, Transactions American Mathematical Society, 1914, pages 227-235; $\mathrm{B}_{2}$ to a paper read before this Society, San Francisco Section, April 6, 1918 (see this Bulletin, 1918, page 418).

* An exposition of the theory of the geometry of numbers is to appear in the Annals of Mathematics during the fall and winter of the present year. For this reason the report here given of the lecture at the Chicago Symposium is very brief. Proofs have been omitted, and only a few illustrative examples are included. The fundamental theorems are, however, stated practically in full (\$3).

$\dagger$ See $B_{1}$, p. 233, for references. 
2. Minkowski's "nowhere concave" surfaces $\left(\mathrm{M}_{2}\right.$, pages 1-76). - It remained for Minkowski to discover a theorem bearing on the least values of a very general class of functions, by means of an elegant geometrical interpretation of this minimum. He defines a real function $\varphi$ of $n$ real finite variables $x_{1}, \cdots, x_{n}$ having the following properties (A), (B), (C):

(A) $\varphi\left(x_{1}, \cdots, x_{n}\right)=$ a definite positive number unless

$$
\begin{array}{ll} 
& \varphi(0, \cdots, 0)=0 ; \quad x_{1}=0, \cdots, x_{n}=0 ; \\
& \varphi\left(t x_{1}, \cdots, t x_{n}\right)=t \varphi\left(x_{1}, \cdots, x_{n}\right) \text { for any positive number } t ; \\
\text { (B) } \varphi\left(x_{1}+y_{1}, \cdots, x_{n}+y_{n}\right) \leqq \varphi\left(x_{1}, \cdots, x_{n}\right)+\varphi\left(y_{1}, \cdots, y_{n}\right) ; \\
\text { (C) } \varphi\left(-x_{1}, \cdots,-x_{n}\right)=\varphi\left(x_{1}, \ldots, x_{n}\right) .
\end{array}
$$

The following functions (1) and (2) may serve as illustrations. We have taken $n=2, x_{1}=x, x_{2}=y$.

$$
\begin{aligned}
& \text { (1) } \varphi=\sqrt{a x^{2}+2 b x y+c y^{2}}, a c-b^{2}>0 ; \\
& \text { (2) } \varphi=|x| \text { when }|x| \geqq|y| ; \varphi=|y| \text { when }|x| \leqq|y| \text {. }
\end{aligned}
$$

The corresponding curves $\varphi=k$ are respectively an ellipse and a square.

It may be proved that

(a) $\varphi$ is continuous in the variables $x_{1}, \cdots, x_{n}$;

(b) the variables satisfying the inequality $\varphi \leqq k$, for a given finite positive $k$, do not exceed in absolute value a certain finite number depending on $k$ and $\varphi$;

(c) the surface $\varphi=k$ has the origin $(0, \ldots, 0)$ as a center of symmetry and is nowhere convex as seen from this center;

(d) for a given positive $k$, the points $\left(x_{1}, \ldots, x_{n}\right)$ are separated into three distinct sets: inner points, points on the surface, outer points, according as the following conditions are satisfied: $\varphi<k, \varphi=k, \varphi>k$;

(e) the outer and inner volumes of $\varphi=k$ have one and the same value, namely the integral $\int d x_{1} d x_{2} \cdots d x_{n}$ extended to all the inner points.

It follows that only a finite number of lattice points, (i. e., points whose coordinates are integers (positive or negative or zero), will be inner points or points on the surface $\varphi=k$, 
and that the surface will lie in the interior of a cube (ndimensional) of finite edge. If $k$ is taken small enough, all lattice points other than the origin will be outer points.

3. General theorems. For a surface as thus defined, Minkowski proved the following theorems I, I', III:

Theorem I ( $\mathrm{M}_{2}$, page 76$)$. If the volume of $\varphi=k i s \geq 2^{n}$, then at least one lattice point other than $(0, \cdots, 0)$ will be an inner point or a point on the surface.

Theorem $\mathrm{I}^{\prime}\left(\mathrm{M}_{2}\right.$, page 76$)$. If the volume of $\varphi=1$ is represented by $J$, then there will be at least one set of integers $l_{1}, \cdots, l_{n}$ not all zero, such that

$$
0<\varphi\left(l_{1}, \cdots, l_{n}\right) \leqq 2 / J^{1 / n} .
$$

The somewhat more general geometrical theorem proved by the author $\left(\mathrm{B}_{1}\right.$, page 228$)$ may be stated as follows in conde nsed form:

TheOREM II. A simple closed surface of volume $V$ can be placed in such a position by means of a translation $x_{1}=x_{1}{ }^{\prime}+a_{1}$, $\cdots, x_{n}=x_{n}{ }^{\prime}+a_{n}$ that the number of lattice points which will lie inside or on the surface is $>V$.

This surface need not possess a center, not does it need to be concave towards an inner point.

Theorem III $\left(\mathrm{M}_{3}\right.$, page 270$)$. There exists a function $\varphi^{\prime}$ obtained from $\varphi$ by means of a linear homogeneous transformation of determinant unity of the variables $x_{1}, \cdots, x_{n}$, such that

$$
\left[\varphi^{\prime}\right] \geqq\left\{\frac{2\left(1+\frac{1}{2^{n}}+\frac{1}{3^{n}}+\cdots\right)}{J}\right\}^{1 / n} .
$$

Geometrically: if the volume of $\varphi^{\prime}=k$ is

$$
<2\left(1+\frac{1}{2^{n}}+\frac{1}{3^{n}}+\ldots\right),
$$

then will every lattice point except $(0, \cdots, 0)$ be an outer point. The author has obtained slightly better results $\left(\mathrm{B}_{2}\right)$.

4. Applications to homogeneous forms.

Theorem IV ( $\mathrm{M}_{1}$, pages 68-79). Let $\xi=\lambda x+\lambda^{\prime} y+\lambda^{\prime \prime} z$, $\eta=\mu x+\cdots, \zeta=\nu x+\cdots$ be three linear forms in $x, y, z$ having real coefficients $\lambda, \cdots, \nu^{\prime \prime}$ and $a$ determinant $\Delta=\left(\lambda \mu^{\prime} \nu^{\prime \prime}\right) \neq 0$. Then 
(a) integers $l_{1}, m_{1}, n_{1}$, not all zero, may be substituted for $x, y, z$, $-\operatorname{say} \xi_{1}=\lambda l_{1}+\lambda^{\prime} m_{1}+\lambda^{\prime \prime} n_{1}, \cdots,-$ such that

$$
\left|\xi_{1}\right|<\sqrt[3]{|\Delta|},\left|\eta_{1}\right|<\sqrt[3]{|\Delta|},\left|\zeta_{1}\right| \leqq \sqrt[3]{|\Delta|}
$$

(b) integers $l_{2}, m_{2}, n_{2}$, not all zero, may be substituted for $x, y$, z such that

$$
\left|\xi_{2}\right|+\left|\eta_{2}\right|+\left|\zeta_{2}\right|<\sqrt[3]{6|\Delta|} .
$$

These results follow immediately from Theorem $\mathrm{I}^{\prime}$, the corresponding surface $\varphi=1$ being a parallelopiped and an octahedron respectively. By a special analysis of the octahedron Minkowski proved $\left(\mathrm{M}_{4}\right.$, page 40$)$ that the left-hand member under (b) is $\leqq \sqrt[3]{\frac{108}{19}}|\bar{\Delta}|$.

From this result we derive $\left|\xi_{2} \eta_{2} \zeta_{2}\right| \leqq \frac{4}{1 \overline{9}}|\Delta|$.

Similar results are obtained when the coefficients of $\xi$ and $\eta$ are conjugate imaginary $(\eta=\bar{\xi})$ and those of $\zeta$ real. Thus we find $\left(\mathrm{B}_{1}\right.$, page 233 , from Theorem III)

$$
\left|\xi_{3} \bar{\xi}_{3} \zeta_{3}\right|<\sqrt{\frac{2}{2}}|\Delta| \text {. }
$$

Theorem V. Let $f$ be a positive definite quadratic form in $n$ variables and of determinant $D$. Then integers, not all zero, may be substituted for the variables such that the numerical value of $f$ is

$$
[f] \leqq \frac{4}{\pi}\left\{\Gamma\left(1+\frac{n}{2}\right)\right\}^{2 / n} D^{1 / n}
$$

The author has obtained the limit

$$
\frac{2}{\pi}\left\{\Gamma\left(1+\frac{n+2}{2}\right)\right\}^{2 / n} D^{1 / n}
$$

by means of Theorem II ( $\mathrm{B}_{1}$, page 233$)$. The exact (superior) limit to the lowest value $[f]$ (i. e., the limit actually reached by at least one quadratic form of determinant $D$ ) has been determined for $n=2,3,4,5$ (see $\mathrm{B}_{1}$, page 233). For any $n$, we know from Theorem III above that this exact limit, which we shall denote by $4(\rho / J)^{2 / n}$, cannot fall below

$$
\frac{1}{\pi}\left\{2\left(1+\frac{1}{2^{n}}+\frac{1}{3^{n}}+\cdots\right) \Gamma\left(1+\frac{n}{2}\right)\right\}^{2 / n} D^{1 / n}
$$

i. e.,

$$
\frac{1+1 / 2^{n}+1 / 3^{n}+\cdots}{2^{n-1}} \leqq \rho \leqq \frac{n / 2+1}{2^{n / 2}} .
$$


Hence, quadratic forms exist for which the corresponding values of $\rho$ satisfy these inequalities. However, no such form has ever been constructed for large values of $n$.

The problem of the closest regular (latticed) packing of spheres in $R_{n}$ is equivalent to the problem of finding that positive definite quadratic form in $n$ variables and of given determinant, say $D=1$, whose least value, other than zero, is the highest possible for the given $n$ and $D\left(\mathrm{M}_{4}\right.$, page $74 \mathrm{ff}$.). The ratio of the space occupied by spheres packed in regular layers in a large cube, say, to the volume of the whole cube, is indeed the number $\rho$ defined above.

The author has recently proved, by an essentially different method, that no matter how the spheres be packed in a large volume $V$, in a "regular" fashion or not, the ratio of the space occupied by the spheres to the whole volume $V$ is

$$
<\frac{n / 2+1}{2^{n / 2}} \text {. }
$$

It may be of interest to note in passing that it follows from the inequalities (1) satisfied by $\rho$ for certain (though unknown) quadratic forms, that the shot-pile packing of spheres, though the closest packing in space of two dimensions and presumably also in space of three dimensions, is very far from being the closest packing in space of a large number of dimensions $\left(\mathrm{M}_{4}\right.$, page 95$)$.

\section{APPLICATIONS OF THE GEOMETRY OF NUMBERS TO ALGEBRAIC NUMBERS.}

BY PROFESSOR L. E. DICKSON.

(Read at the Chicago Symposium of the American Mathematical Society, March 28, 1919.)

1. The geometry of numbers not only furnishes a concrete geometric image of certain fundamental theorems on algebraic numbers, but also provides a new and attractive method of proving important theorems on algebraic fields. For the sake of concreteness we shall restrict attention to the typical case of the cubic field $F(\theta)$, which is composed of the numbers 